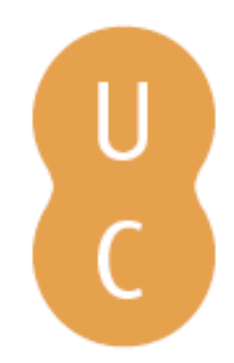

\title{
pommalina
}

\section{Una nueva definición de la catalogación a partir de los principios internacionales de catalogación}

\author{
Autor(es): $\quad$ Picco, Paola \\ Publicado por: Imprensa da Universidade de Coimbra \\ URL \\ persistente: URI:http://hdl.handle.net/10316.2/31914 \\ DOI: $\quad$ DOI:http://dx.doi.org/10.14195/978-989-26-0869-3_17 \\ Accessed : $\quad$ 26-Apr-2023 13:01:47
}

A navegação consulta e descarregamento dos títulos inseridos nas Bibliotecas Digitais UC Digitalis, UC Pombalina e UC Impactum, pressupõem a aceitação plena e sem reservas dos Termos e Condições de Uso destas Bibliotecas Digitais, disponíveis em https://digitalis.uc.pt/pt-pt/termos.

Conforme exposto nos referidos Termos e Condições de Uso, o descarregamento de títulos de acesso restrito requer uma licença válida de autorização devendo o utilizador aceder ao(s) documento(s) a partir de um endereço de IP da instituição detentora da supramencionada licença.

Ao utilizador é apenas permitido o descarregamento para uso pessoal, pelo que o emprego do(s) título(s) descarregado(s) para outro fim, designadamente comercial, carece de autorização do respetivo autor ou editor da obra.

Na medida em que todas as obras da UC Digitalis se encontram protegidas pelo Código do Direito de Autor e Direitos Conexos e demais legislação aplicável, toda a cópia, parcial ou total, deste documento, nos casos em que é legalmente admitida, deverá conter ou fazer-se acompanhar por este aviso.

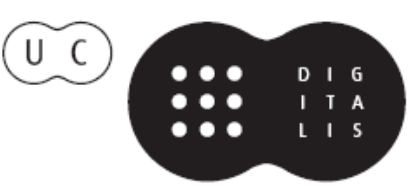


Maria Manuel Borges

Elias Sanz Casado

Coordenação

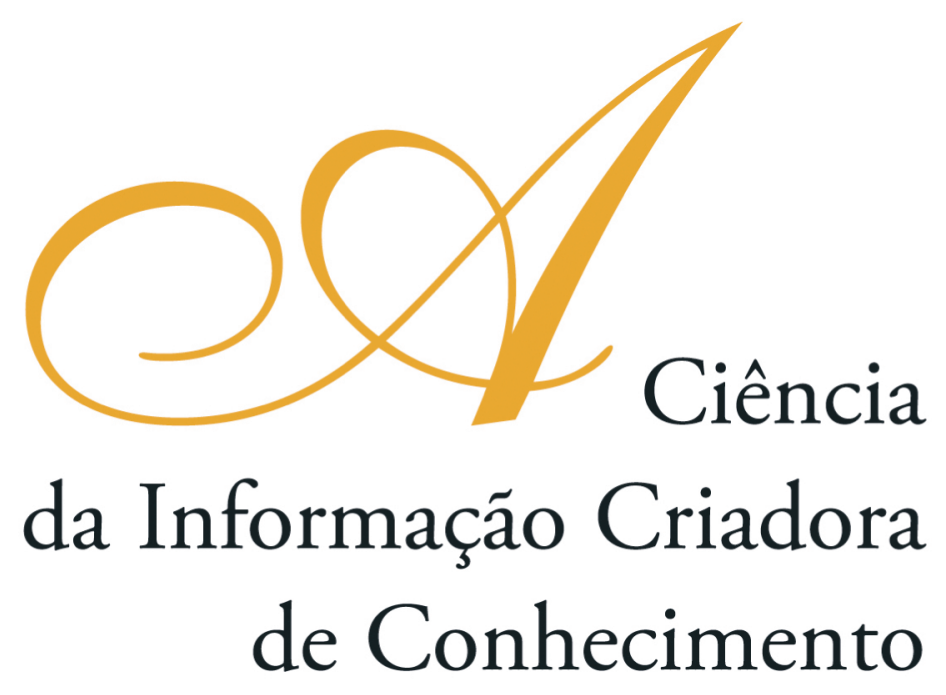

Vol. I I

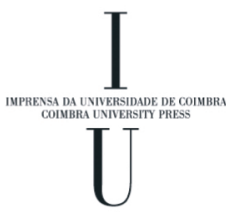

- COImbra 2009 


\title{
UNA NUEVA DEFINICIÓN DE LA CATALOgACIÓN A PARTIR de los Principios Internacionales de Catalogación
}

\author{
Paola Picco \\ Universidad de la República (Uruguai)
}

\section{Resumen}

El vertiginoso desarrollo de las Tecnologías de la Información y la Comunicación y su rápida acogida por las bibliotecas y especialmente el impacto que han tenido en el proceso de catalogación han hecho reformular muchos de los principios que sostenían esta práctica fundamental para cualquier biblioteca. El primer intento por normalizar esta práctica se remonta a la década del 60 con la Conferencia de París donde se definieron una serie de principios que fueron el sustento de la mayoría de los Códigos de Catalogación hasta la actualidad. Pasados más de 40 años se vio la necesidad de actualizar los mismos. Las bibliotecas, los soportes de información, las prácticas editoriales y hasta los propios usuarios han cambiado. Es así que en el año 2003 se comienza a trabajar en una nueva Declaración de Principios Internacionales de Catalogación con el objeto de contribuir a solucionar los vacios existentes y que estos principios fueran el sustento de un futuro Código de Catalogación Internacional.

En este marco es interesante destacar que los nuevos Principios Internacionales de Catalogación fueron pensados a partir de las necesidades de los usuarios. Es así que se definen las actividades o tareas que el usuario realiza en el catálogo cada vez que necesita buscar información. Este es un elemento central, porque ubica al usuario como el objetivo fundamental de la catalogación.

Es en este contexto que se propone una definición de la catalogación, partiendo de las definiciones tradicionales que centra a la catalogación como la descripción de los documentos, arribando a una nueva definición que contemple a la misma como el proceso por el cual se describen a las distintas entidades que conforman un recurso bibliográfico. En este proceso es fundamental identificar cada una de estas identidades: la obra y su expresión y describir las características de su manifestación y del ítem y sus relaciones como forma de satisfacer las necesidades del usuario. .

Se destaca que a partir de esta nueva definición de la catalogación, los catálogos se convierten en poderosas herramientas de recuperación de información y pasan a ser un elemento central en el desarrollo y fomento de la producción de nuevo conocimiento.

El objetivo de esta presentación es demostrar como a partir de la incorporación de los Principios Internacionales de Catalogación la catalogación deja de visualizarse como una mera técnica para convertirse en un proceso fundamental en el fomento de la producción científica y tecnológica de cualquier sociedad.

Como metodología se utiliza la revisión bibliográfica para identificar distintas definiciones de catalogación propuestas por diferentes autores. A partir de esta identificación se procede a la ubicación de las mismas en los distintos paradigmas disciplinares. Se analizan los resultados obtenidos y a partir del análisis del nuevo contexto ya descrito y de las definiciones analizadas se procede a proponer una nueva definición. La misma incorpora la visión de los Principios Internacionales de Catalogación y se define como una actividad fundamental para mediar entre el usuario y el conocimiento. Asimismo se propone presentar a la catalogación como una actividad fundamental en la generación de nuevo conocimiento. 


\section{Abstract}

The development and application of information and communication technologies, its adaptation to libraries'needs, and the impact over cataloging process have contributed to reformulate some of the principles which founded this fundamental practice for any library. The first attempt to normalize this practice was in the 60's at the Paris Conference where a series of Principles were agreed. They were assumed as the basis of many Cataloging Codes till today. The libraries, the document formats, the editorial practices and the users have changed. It is in this way, that in 2003 opened a new era for the discussion of new cataloging principles among the international community which were in coherence with the cataloging practices.

Thus, it is interesting to enhance that the new International Cataloging Principles were defined taking into account the users needs and their tasks with regards to the catalog. The users become themselves into the main focus of this activity.

It is in this context, that a cataloging definition is proposed. This study begins analyzing traditional definitions which focus cataloging on the document description, it is arrived to a new one which states that cataloging is a process where the entities which form a resource are described. It is fundamental identify each one: work, expression, manifestation and item. It also requires the establishment of entities'relationships as a way to satisfy users' needs.

Since the proposed definition, catalogs become into powerful tools, capable of retrieving information and come to be a main issue in the creation of knowledge.

The objective of this article is to show how the Declaration of International Principles of Cataloging have affected the definition of this discipline, changing from being a simple technique to become into a tool of scientific and technological promotion.

The methodology used is the bibliographic review which allowed the identification of different cataloging definitions proposed by several authors.

These definitions are analyzed in the light of different disciplinary paradigms. The results are analyzed and a new definition is proposed. It incorporated the vision of the Declaration of the International Principles of Cataloging and the discipline is defined as essential in the relationship knowledge-users. It is stated that cataloging is related with knowledge generation.

\section{Introducción}

La catalogación comienza a utilizarse en las antiguas bibliotecas como una técnica que permitía establecer el control patrimonial de las distintas colecciones. En la literatura sobre el tema se encuentran referencias a los catálogos de aquella época como registros patrimoniales donde se listaban las existencias de estas unidades (Garrido, Arilla, 1999, p.62). En todo caso, esos catálogos no respondían a los objetivos de los catálogos actuales que actúan como herramientas de organización y recuperación de la información y del conocimiento.

La catalogación se ha desarrollado como técnica y se ha convertido en una disciplina con un cuerpo teórico y una serie de principios que permiten comprender el alcance y objetivos de la misma. La evolución en la concepción de las bibliotecas, su misión y objetivos, así como las colecciones y sus distintos soportes han obligado a repensar la catalogación en este nuevo contexto.

Esta disciplina estuvo tradicionalmente pensada como una herramienta que facilitara la tarea del catalogador, dejando en un segundo plano las necesidades del usuario. En este sentido la catalogación avanzó principalmente basada en la casuística y en la resolución de problemas y la teoría trató de acompasar y explicar las soluciones propuestas. 
Fue así Seymour Lubetzky en la década del 60 quien plantea por primera vez la necesidad de desarrollar códigos y prácticas de catalogación basadas en principios, abandonando así la tendencia a establecer múltiples reglas que contemplaran los distintos casos que se presentaban en la realidad. Esa situación traía aparejada un extenso número de reglas e inconsistencias en su aplicación. Podemos citar como ejemplos las 369 reglas de Cutter y las 91 de Panizzi y la dificultad de conciliar las prácticas catalográficas a nivel internacional. Se puede mencionar como paradigmático el caso de la Reglas Angloamericanas en su primera edición y la imposibilidad de acordar un único texto para los dos países involucrados en su redacción: Estados Unidos e Inglaterra. Esta situación recién se pudo revertir luego de la aprobación de los Principios Internacionales de Paris y de las ISBD en 1961 y 1969 respectivamente y con la edición de la segunda edición de las Reglas de Catalogación Angloamericanas en 1978.

Tanto los Principios Internacionales de París, como la aprobación y adopción de las ISBD, han hecho importantes contribuciones a la normalización. La mayoría de los códigos de catalogación a nivel internacional han incorporado estos principios y normas en sus sustentos teóricos. Pero pasados más de 40 años de su aprobación y con el impacto que ha tenido la incorporación de las tecnologías de la información tanto a las actividades de gestión bibliotecaria como a la producción de información, estos han quedado desactualizados, y una vez más se puede afirmar que la catalogación avanza como disciplina práctica y luego se desarrolla un cuerpo teórico que trata de fundamentar su aplicación.

En la década de los 90 la IFLA y la Library of Congress preocupadas por los avances tecnológicos y la brecha que se estaba generando con las prácticas catalográficas tradicionales abogan por la definición de un marco conceptual que permita simplificar esta práctica. Es así que luego de varios años de reuniones internacionales entre expertos se aprueba en el año 1997 el modelo Requisitos Funcionales para los Registros Bibliográficos (FRBR). Por primera vez en la historia de esta disciplina se cuenta con un modelo conceptual que esta pensado para representar el universo bibliográfico tal cual existe en la realidad. Para ello, este modelo define tres grupos de entidades y sus correspondientes atributos que permiten realizar la descripción y establecer relaciones entre las distintas entidades. El modelo está pensado desde las necesidades de los usuarios, otro cambio a destacar en esta nueva concepción de la catalogación.

Por otro lado, la comunidad bibliotecológica a nivel internacional empieza en el año 2003 a analizar un conjunto de principios que le den sustento a las nuevas prácticas que se vienen desarrollando en las bibliotecas y unidades de información y que incorpore además el marco conceptual FRBR aprobado a nivel internacional. La evolución del catálogo en fichas, en primer lugar a las bases de datos, y luego a los OPAC (catálogo en línea de acceso público) ha hecho más que necesario que se acuerden un conjunto de elementos que le dieran coherencia y consistencia a estos desarrollos. A partir del desarrollo de los OPAC, se sintió cada vez más la necesidad de normalizar las prácticas. El desarrollo de estos catálogos permitieron las consultas remotas, a varios catálogos a la vez, el intercambio de registros bibliográficos a través de protocolos de comunicación, el desarrollo de catálogos colectivos a nivel internacional. Hechos muy significativos que han revolucionado la concepción de los catálogos tradicionales y de las propias bibliotecas. Fue entonces el momento de potenciar estos usos, y acordar 
principios que permitieran el desarrollo de estas poderosas herramientas como forma de promocionar la generación y el acceso al conocimiento.

Es en este contexto, que se pretende abordar la función y definición de la catalogación. La Declaración de Principios Internacionales de Catalogación aprobados en el año 2009, piensan a la disciplina desde la perspectiva de los intereses del usuario, abandonando completamente la visión de registro patrimonial que tuvo la misma en su inicio. Esta Declaración, pretende sustituir y ampliar el alcance de los Principios de Paris, de 1961 e incluir todo tipo de materiales, asimismo pretende abarcar a "todos los aspectos de los datos bibliográficos y de autoridad”. (IFLA, 2009, p. 1). Define cinco funciones básicas a las que debe responder cualquier catálogo: en primer lugar, el catálogo deberá permitir a un usuario encontrar cualquier recurso bibliográfico como resultado de una búsqueda, esta función puede implicar además encontrar conjuntos de recursos que representen a una misma obra, una misma expresión, etc. En segundo lugar deberá permitir que el usuario identifique el recurso, significa que a través de la información proporcionada, el usuario determine si es lo que estaba buscando. En tercer lugar seleccionar un recurso de acuerdo a sus necesidades, la cuarta función sería adquirir u obtener y esto tiene que ver con el acceso directo a ese recurso a través de la ubicación física o virtual y la última función tiene que ver con la posibilidad de navegar en el catálogo a través de las distintas relaciones que se establecen aplicando el modelo entidad relación. (IFLA, 2009, p.3, 4)

De esta manera, el objetivo de la presente ponencia es demostrar como a partir de la incorporación de los Principios Internacionales de Catalogación, la catalogación se convierte en un proceso fundamental en el fomento de la producción científica y tecnológica de cualquier sociedad y cómo la definición de la disciplina debe ser actualizada.

Al respecto López Guillamón sostiene

[...] la catalogación está siendo especial objeto de estudio como función esencial en la gestión y organización del conocimiento y como operación inserta en el engranaje de actuación de una biblioteca, entendida en su sentido más abstracto. En una sociedad en la que día a día se valora más la ciencia y la tecnología como motores de desarrollo, parece que la catalogación es un buen agente en el proceso mismo de hacer ciencia y tecnología. (López Guillamón, 2004, p.194)

La catalogación y su relación con las Ciencias de la Documentación, la Ciencia de la Información y la Gestión del Conocimiento.

Antes de avanzar con este estudio, se hace necesario, explicar como la Catalogación es una disciplina que se puede ubicar tanto en las Ciencias de la Documentación, como en la Ciencia de la Información y en la propia Gestión del Conocimiento. A lo largo de los últimos años, en la literatura se pueden encontrar distintos trabajos que tratan de explicar los alcances y diferencias de cada una de ellas. Los mencionados trabajos reflejan la dificultad de definir los límites de una y de otras y de explicar los elementos que tienen en común y aquellos que las distinguen. En algunos casos, las diferencias están marcadas por las tradiciones de los países donde surgen y se desarrollan, más que por los alcances mismos de cada una. En otros casos, por el rol que ocupa el profesional y su relación con la sociedad. De todas maneras, si se puede coincidir en que ha existido una constante evolución, signada por la importancia que ha ido adquiriendo la 
información como elemento característico de la estructura social imperante. Los cambios tecnológicos, los beneficios que se obtienen a partir del acceso a la información y al conocimiento, el valor económico que ha adquirido la producción de bienes vinculados a la información, y el conocimiento en si mismo, como noción de promoción del desarrollo de la sociedad han marcado la evolución de estas disciplinas.

Se pretende entonces, definir a las Ciencias de la Documentación, a la Ciencia de la Información y la Gestión del Conocimiento para posteriormente relacionar a la catalogación con las mismas, y observar como varía la definición y función de la catalogación en relación con cada una de ellas. Se busca de esta manera, a partir de la Declaración Internacional de los Principios de Catalogación ubicar a la Catalogación dentro de la disciplina más coherente con las funciones y objetivos definidos a partir de la mencionada declaración.

Como se mencionó anteriormente, existen múltiples definiciones respecto a estas disciplinas e incluso sobre su denominación, en esta oportunidad no se va a entrar en la discusión de cual sería la más adecuada, simplemente se va a tomar como definiciones aceptadas las que se transcriben a continuación:

\section{López Yepes sostiene que:}

El objeto de estudio de la Documentación se basa, a nuestro entender, en el concepto de información documental y en el concepto de proceso documental como un proceso informativo y de naturaleza peculiar por cuando se da en el mismo una actividad de recuperación de mensajes informativos emitidos en procesos anteriores y que, mediante su conserva y tratamiento técnico, se difunden transformados con la finalidad de que sirvan de fuente de información para la obtención de nuevo conocimiento o para la acertada toma de decisiones en las organizaciones, empresas e instituciones. (López Yepes , 2006, p. 70)

\section{El mismo autor agrega}

Como Ciencias de la Documentación, esto es, como el conjunto de las disciplinas que tiene por objeto de estudio de un proceso informativo en el que se da una actividad de recuperación de mensajes emitidos en procesos anteriores y que, mediante análisis y tratamiento técnico, se comunican transformados con la finalidad de que sirvan de fuente de información para la obtención de nuevo conocimiento para la toma de decisiones. (López Yepes, 2006, p.71)

Pérez Pullido y Herrera Morillas explican como a partir de la década de los 60 adquiere particular relevancia el concepto de información frente al de soporte o documento. Explican así la aparición de la Ciencia de la Información y sostienen que "se sustenta en la adaptación a las necesidades de acceso a la información que la ciencia demanda, lo que Shera denomina el principio de acceso al saber registrado". Agregan los autores "se considera una ciencia interdisciplinar.... La teórica, relacionada con otras ciencias como las matemáticas, la ligüística o la psicología, y la aplicada, relacionada con la recuperación, organización y difusión de la información" (Pérez Pullido, Herrera Morillas, 2006, p.61) 
Por otro lado Davis, explica como en la sociedad occidental se está transitando de la Era de la Información a la Era de la Mente "Age of the Mind" o Sociedad del Conocimiento. Esta transición esta marcada por la aplicación de las tecnologías y ha dado lugar "a la organización basada en el conocimiento" (Riley, 1998, citado por DAvis, p.69) Agrega esta autora: "Con el advenimiento del capitalismo informacional, el foco de la Bibliotecología o de la Ciencia de la Información ha cambiado hacia la gestión de la información” (Davis, 2008, p. 69). Continuando con su explicación, la mencionada autora cita a Muddiman quien afirma que:

Los fundamentos de la Bibliotecología tradicional, que se sostenía sobre la necesidad de difundir el conocimiento público para generar el progreso y el bien general, esta siendo sustituida por la tendencia hacia la gestión de la información donde el compromiso de una profesión de servicio es remplazada por el compromiso hacia una economía de mercado (Muddiman, 1999, citado por Davis, 2008, p. 69).

Esta misma autora concluye en que la Gestión de la Información termina dando lugar a la Gestión del Conocimiento y cita nuevamente a Muddiman quien sostiene que el objetivo de la Gestión del Conocimiento es "la explotación de las entidades informativas en una organización con el fin de utilizar la información para lograr valor agregado y obtener ventajas competitivas" (Muddiman, 1999 citado por Davis, 2008, p.69)

\section{Metodología}

Para alcanzar el objetivo definido para esta investigación, se procede al análisis de distintas definiciones sobre catalogación propuestas en la literatura, se comparan, se analizan, se contextualizan y se propone una nueva a partir de los Principios Internacionales de Catalogación que permiten comprender el valor de la misma en la promoción y generación de conocimiento.

Se parte de un relevamiento bibliográfico de artículos sobre la temática que permitió identificar distintas definiciones. Se aplica como método de estudio, el análisis de contenido, con el cual se realiza una evaluación cualitativa entre las distintas definiciones identificadas, donde luego se procede a caracterizar los aspectos coincidentes y divergentes. El análisis de contenido se define como una técnica utilizada en las ciencias sociales y humanas que permite “... analizar los documentos no cifrados. Se puede limitar a analizar el contenido manifiesto de un documento, lo que se dice explícitamente, o se puede llegar a realizar una interpretación para analizar su contenido subyacente. El análisis de contenido permite estudiar la simbología de las palabras, frases, ... y de comparar las producciones de grupo o individuos diferentes, hacer un estudio en el tiempo y enriquecer el fenómeno estudiado con diversas explicaciones. Por oposición el análisis de contenido exige que se disponga de suficiente tiempo para decorticar cada uno de los documentos ..." (Angers, 2000, p. 81) ${ }^{1}$

\footnotetext{
1 Traducción libre del francés.
} 
En esta oportunidad se va a trabajar sobre definiciones de catalogación propuestas por distintos autores, se va a proceder al análisis de las mismas identificando similitudes y divergencias y se va a contextualizar su significado. Se toman como referencia para este estudio las siguientes definiciones:

Catalogación: Representación normalizada de los datos documentales para la localización física de los documentos ordenados en catálogos según criterios determinados (Reglas de catalogación). La catalogación es un proceso técnico que tiene por fin sistematizar el conjunto bibliográfico de acuerdo con normas para darle unidad y coherencia. Comprende dos pasos: primero, la redacción del asiento bibliográfico, y segundo, la formación del catálogo mediante la ordenación de aquellos. (de Sousa, 2004, p.156)

La catalogación descriptiva es la fase del proceso de catalogación que implica la identificación y descripción de un ítem, el registro de esta información en la forma de un registro catalográfico y la selección y formación de puntos de acceso, con la excepción de los puntos de acceso temático. La catalogación descriptiva toma los aspectos físicos de un ítem e identifica la responsabilidad intelectual de su contenido, sin hacer referencia a la clasificación temática o a la asignación de encabezamientos temáticos, correspondiendo estos dos últimos a aspectos de la catalogación temática. (Taylor, 1992, p.18) ${ }^{2}$

...la catalogación como todo un proceso unitario, que partiendo de la descripción del documento (Descripción bibliográfica), pasa por la elección de puntos de acceso (personales, corporativos, títulos, temáticos y sistemáticos) y contempla, a nivel de asiento, con la formación y transcripción de los datos locales (signatura y registro) (Garrido Arilla, 1999, p.25)

La catalogación es el proceso por el que se describe un recurso bibliográfico, se identifica la/s obra/s que contiene, y se determina qué elementos de la descripción y de la obra constituirán puntos de acceso en un catálogo. Implica la recolección de datos y su transcripción según normas estandarizadas, de modo que el resultado sea uniforme y por lo tanto más fácilmente interpretable. El objetivo de la catalogación es recoger de aquellos datos que permitan la identificación de un recurso, su distinción de otros recursos u obras y la elección por parte de un usuario de un recurso u otro a partir de su descripción. (Spedalieri, 2007, p. 24)

\section{Discusión de los resultados.}

Del relevamiento realizado sobre definiciones de catalogación, se observa que no existe un consenso sobre el alcance de la disciplina, sucede algo similar con las definiciones de las otras tres disciplinas utilizadas como referencia para este

\footnotetext{
2 Traducción libre del inglés
} 
trabajo: Ciencias de la Documentación, Ciencia de la Información y Gestión del Conocimiento.

En primer lugar se puede afirmar que, la Catalogación se puede considerar como una disciplina auxiliar de cualquiera de las tres anteriormente mencionadas. Se puede observar en las definiciones de estas tres disciplinas, que los autores coinciden en señalar el corte interdisciplinario que tiene cualquiera de ellas. Al respecto seńalaba López Yepes (2006) "Como Ciencias de la Documentación, esto es, como el conjunto de las disciplinas que tiene por objeto de estudio de un proceso informativo ..." y Pérez Pullido y Herrera Morillas (2006), haciendo referencia a la Ciencia de la Información, sostienen que "se considera una ciencia interdisciplinar ...". Si bien la definición sobre Gestión del Conocimiento no hace referencia a la confluencia de las disciplinas, se puede observar por la complejidad del alcance, la necesidad de hacer uso de múltiples intervenciones desde distintas ópticas.

Si se parte de la hipótesis de que la catalogación puede ser una disciplina que hace parte de las anteriormente mencionadas o que es auxiliar de las mismas, se puede sostener entonces que las distintas definiciones sobre catalogación están vinculadas a estas disciplinas mayores.

Si se observa la primera definición retenida, la misma alude a "datos documentales para su localización", y a "documentos ordenados en catálogos según criterios determinados" . En el segundo caso, se hace referencia a la "identificación y descripción de un ítem" y a la "selección y formación de puntos de acceso", en el tercer caso a la "descripción de un documento" y a la "elección de puntos de acceso". Y la última a la descripción de "un recurso bibliográfico, la identificación de la obra que contiene ..." y a los "puntos de acceso" también menciona la necesidad de "...recoger datos que permitan la identificación de un recurso, su distinción de otros recursos u obras y la elección por parte de un usuario de un recurso u otro..."

Es interesante destacar como las tres primeras definiciones hacen énfasis en la descripción de los aspectos documentales (documento, ítem), por oposición a la última que se refiere al recurso bibliográfico.

En segundo lugar, se observa que la primera definición se centra en la localización y ordenación, mientras que las restantes coinciden en que la catalogación se ocupa de la descripción, mientras que dos definiciones, mencionan además a la identificación. Se debe destacar que solamente una definición recoge la necesidad de brindar elementos para que el usuario pueda realizar la "elección" , entiéndase la correcta elección, o la elección de acuerdo a sus intereses.

En el caso de las tres primeras definiciones, se observa un fuerte énfasis en la descripción o registro de los aspectos externos, por dicha razón hacen referencia al documento o al ítem. En el caso de la definición de Taylor, expresa claramente esta situación haciendo énfasis en que la catalogación descriptiva "toma los aspectos físicos de un ítem" y cuando se refiere al contenido aclara que solamente se "identifica la responsabilidad intelectual de su contenido" Esta definición distingue claramente la catalogación descriptiva de la catalogación temática. Entiéndase por esta última el proceso de asignación de puntos de accesos temáticos.

Sin embargo, Spedalieri, siguiendo con la catalogación descriptiva, manifiesta la necesidad de identificar el contenido del recurso bibliográfico, aludiendo en este caso a la obra. La definición de Spedalieri, introduce la noción de registrar el contenido 
en la catalogación descriptiva, por oposición a los otros autores que hacen un fuerte énfasis en los aspectos externos o físicos. Se debe entender entonces, el alcance del término obra, y por ende establecer su relación con el soporte o continente que es el documento. En este sentido, el modelo FRBR y la definición de las entidades pueden asistir a entender la posición de la autora.

Para explicar esta definición, se utilizará el primer grupo de las entidades definidas por el modelo FRBR que incluyen a la obra, la expresión, la manifestación y el ítem. Como se mencionó anteriormente, este modelo representa al universo bibliográfico tal cual existe en la realidad. Por lo tanto se pueden identificar a cada una de las entidades en cualquier recurso bibliográfico que se va a catalogar. Dentro del primer grupo de entidades del modelo FRBR, se definieron dos de carácter abstractas que están directamente relacionadas con el contenido y la forma de expresar el mismo, y dos entidades que son las que permiten materializar ese contenido que se expresa de determinada forma y que están representados por la manifestación y el ítem.

El modelo FRBR define a la obra como la creación intelectual o artística, es lo que el creador imaginó, lo que permanece en la mente de su creador hasta que es expresado de alguna manera. En este sentido, la segunda entidad de este modelo (expresión), define la forma en que se expresa esa creación (que puede ser de forma alfanumérica, notaciones musicales, texto, texto en distintos idiomas, etc.)

Esa creación intelectual, se va a materializar o se va a presentar de alguna forma para que llegue al usuario final, podrá ser así un libro, un CD, un DVD, un archivo digital, lo que corrientemente llamamos documento. Esta entidad es la manifestación, que está directamente relacionada con la forma de presentación de ese contenido y por último el ítem, que es un ejemplar de esa manifestación que se podrá tener en la colección de una biblioteca determinada y que presentará características particulares.

Cabe destacar entonces, el aporte interesante que plantea Spedalieri al referirse a la necesidad de identificar la obra u obras que contiene ese recurso. Esta autora representa muy bien la relación que se da entre las entidades porque establece que se describe un recurso bibliográfico, entiéndase este como el soporte, y se identifica su contenido. El hecho de hacer énfasis en el contenido, tiene directa relación con la inquietud actual de la catalogación de pensar en las necesidades de los usuarios, y de cambiar el alcance de la catalogación de la mera descripción de elementos externos a contemplar también los aspectos internos.

Si se piensa en el usuario, y cómo él se dirige a la biblioteca, generalmente va en busca de un contenido determinado, por ejemplo, una novela como puede ser "Cien años de soledad” de Grabriel García Márquez, o en una ópera de Mozart como "Las Bodas de Fígaro". En ambos casos el usuario está pensando en una creación intelectual o artística determinada. Una vez enfrentado al catálogo, podrá encontrar que la biblioteca tiene la primera edición de Cien años de soledad publicada por Sudamericana, o la edición que conmemora los 40 años de la primera edición u otras ediciones que pueden ser de interés para el usuario. Con respecto al segundo caso, el usuario podrá encontrar que existen distintas expresiones de esa obra, podrá encontrar la partitura de las Bodas de Fígaro, una interpretación determinada, etc. Los ejemplos anteriores ilustran el comportamiento de un usuario cuando se dirige a la biblioteca, y demuestra como para él la prioridad es el contenido. Una vez que ubica el mismo, procede a la selección de acuerdo a otros criterios, que podrán ser la expresión (textual, lengua, 
sonoro, etc.) y a las características de presentación o manifestación (para el caso de una novela podrá ser determinada edición, podrá ser un libro, un CD, etc.)

Es el momento entonces de establecer la relación entre las definiciones propuestas de catalogación y los Principios Internacionales de Catalogación. Estos últimos no definen a la disciplina en si misma, determinan una serie de elementos que se deben de tener en cuenta en la elaboración de los Códigos de Catalogación y en las prácticas mismas para alcanzar productos normalizados y coherentes.

Sin embargo, la aplicación de determinadas pautas, principios, reglas, tienen mayor sentido cuando se sabe a ciencia cierta que es lo qué se busca, o se define claramente qué es lo que se está haciendo. Es por esta razón que se sostiene la necesidad de contar con una definición coherente con los nuevos principios de catalogación.

La definición propuesta por Spedalieri es la que más se acerca a los Principios Internacionales de Catalogación, ya que establece la necesidad de establecer la relación entre el recurso bibliográfico y su contenido a través de la identificación de la obra. Toma además algunas de las funciones que se establecen en la Declaración de los Principios Internacionales de Catalogación "identificación", se refiere a la distinción entre recursos que podría asimilarse a la necesidad de que el catálogo brinde elementos para facilitar al usuario la identificación y menciona además la elección. Esta autora en su definición cita al menos tres de las funciones que debe cumplir el catálogo según los mencionados principios (descripción, identificación y selección).

Si bien la definición de Spedalieri, tiene una gran afinidad con lo que se propone desde la Declaración de Principios Internacionales de Catalogación, no enfatiza en la necesidad de establecer las relaciones necesarias entre las distintas entidades definidas.

Se propone entonces como definición de catalogación: el proceso intelectual que requiere el análisis y la identificación de las entidades presentes en los recursos de información, la descripción de cada una de ellas a través de sus atributos, y la asignación de puntos de acceso que permitan al usuario encontrar la entidad o entidades requeridas. Asimismo, este proceso permitirá que el usuario cuente con todos los elementos necesarios para la identificación, selección y acceso a los recursos solicitados. Este proceso permitirá además establecer relaciones entre las distintas entidades para facilitar la navegación del catálogo con el objetivo de favorecer la recuperación, identificación y selección del usuario. Para el registro de las mencionadas entidades se aplicarán estándares aceptados y de fácil comprensión.

Es en este nuevo contexto, donde se asocia a la Catalogación directamente con las necesidades de los usuarios, que se propone esta nueva definición, que toma en cuenta las entidades que se pueden identificar en un recurso y por lo tanto contempla los aspectos intelectuales (contenido) de los mismos. Se destaca que tanto el modelo FRBR como la Declaración de los Principios Internacionales de Catalogación han hecho una contribución importante en este sentido. Evidentemente que esta nueva situación, deja muy bien ubicada a la Catalogación como una disciplina que tiene aportes que realizar al desarrollo de la llamada Sociedad del Conocimiento.

La Catalogación al desprenderse de su fuerte énfasis en los aspectos físicos o externos como se observó en algunas definiciones de referencia para este estudio, y al situar al contenido o a la elaboración intelectual como elemento fundamental para el usuario, pasa a desempeńar una función importante en la generación de nuevo conocimiento. Es el acceso a ese conocimiento registrado, el que va a promocionar la 
reflexión, el análisis, el debate, la confrontación y en definitiva la generación de nuevo conocimiento, elemento de vital importancia en el contexto social actual.

De esta forma se puede fundamentar la capacidad que tiene esta disciplina de adecuarse a los distintos contextos sociales y de aplicación dentro de los distintos paradigmas disciplinares. En la llamada Ciencias de la Documentación, asistió a la descripción y recuperación de los documentos, con un fuerte énfasis en lo externo. En el caso de la Ciencia de la Información el énfasis debió haber estado más en la información registrada que en lo externo, pero es valiosa la opinión de Van House y Sutton al respecto, quienes sostienen que la Ciencia de la Información es una especie en extinción dado que "el foco tradicional de la Ciencia de la Información no ha estado en la información sino en sus contenedores" (Van House and Sutton citado por Davis, 2008, p.67) Davis en su trabajo cita una expresión muy gráfica de Barlow que sostiene "por muchos ańos hemos pensando que estábamos en el negocio de vinos. De hecho nos estábamos dedicando al negocio de embotellar. Y la realidad nos dice que no sabemos nada sobre el vino" (Barlow citado por Davis, p.67).

Si ubicamos entonces a la catalogación dentro de la Gestión del Conocimiento, donde la producción del mismo se ha convertido en una mercancía, o como un elemento promotor del desarrollo científico y tecnológico, es evidente que esta disciplina tiene mucho que aportar. En este sentido, se coincide con la afirmación de López Guillamón de que "la catalogación está siendo especial objeto de estudio como función esencial en la gestión y organización del conocimiento”. (López Guillamón, 2004 , p. 194). Es obvio que para llegar a cumplir con este objetivo, la catalogación debe dejar de ejercer su énfasis sobre los aspectos físicos o de presentación, y ocuparse de los aspectos intrínsecos de los mismos.

En este nuevo escenario, interesa en particular encontrar, identificar, seleccionar y acceder al conocimiento registrado, perdiendo importancia los aspectos físicos o de presentación vinculados a ellos. Será ese conocimiento registrado el que permitirá al usuario reflexionar, pensar, y generar nuevo conocimiento a partir de su utilización.

Es así que se concluye destacando la importancia de la catalogación como herramienta fundamental en promover el acceso y organización del conocimiento registrado y como motor esencial de cualquier sociedad en la promoción del desarrollo científico y tecnológico.

\section{Consideraciones finales}

Se destaca la necesidad de contar con una nueva definición que permita comprender la aplicación de la disciplina en el escenario actual.

La catalogación es una herramienta que ha estado asociada desde la antigüedad a las prácticas de las bibliotecas, asimismo, atraviesa a varias disciplinas como se pudo observar en este análisis como son las Ciencias de la Documentación, la Ciencia de la Información y la Gestión del Conocimiento. Demuestra que ha tenido la versatilidad y flexibilidad de persistir en el tiempo y adaptarse a los requerimientos de varias disciplinas.

En el nuevo escenario mundial, donde la generación de nuevo conocimiento adquiere un valor relevante, puede adaptarse nuevamente, para contribuir en este sentido, 
superando las viejas fronteras de herramienta utilizada o vinculada exclusivamente al campo de acción de las bibliotecas.

Los profesionales de la información, los bibliotecólogos, los catalogadores tienen la expertise y el conocimiento de muchos años de aplicación de los principios de esta disciplina. En este sentido, deben actualizar y compatibilizar los conocimientos con las necesidades y prácticas imperantes y explotar este campo que se presenta actualmente y en futuro próximo como muy promisorio.

Por último, se considera fundamental que la catalogación se ponga al servicio de la Gestión del Conocimiento, pero no se comparte la opinión de Muddiman que sostiene que existe una mutación en la profesión, por una orientación de servicio, hacia un compromiso con una economía de mercado. Se sostiene, que como profesionales de la información y el conocimiento, se debe abogar cada vez más por la necesidad de que todos los individuos puedan acceder al conocimiento de manera equitativa como forma de combatir las inequidades sociales existentes.

\section{Referencias bibliográficas}

ANGERS, M. (2000). Initiation pratique à la methodologie des sciences humaines. Anjou: CEC.

DAVIS, C. (2008). Librarianship in the $21^{\text {st }}$ century-crisis or transformation?. Public Library Quarterly, 27, 57-82.

DE SOUSA, J. M. (2004). Diccionario de Bibliología y Ciencias Afines. 3a edición corregida y aumentada. Gijón: Trea, 2004.

GARRIDO ARILLA, M. R. (1999). Teoría e historia de la catalogación de documentos. Madrid Síntesis, 1999.

IFLA (2009). Declaración de principios internacionales de catalogación. Disponible en: http:// www.ifla.org/files/cataloguing/icp/icp_2009-es.pdf

LÓPEZ GUILLAMÓN, I. (2004). Una nueva catalogación: objetivos, principios, características y control de autoridades. Rev. Esp. Doc. Cient., 27, 192-211.

LOPEZ YEPES, J. Documentación. En: Manual de Ciencias de la Documentación, coordinador José López Yepes. 2a ed. Madrid: Pirámide, 2006.

PEREZ PULIDO, M, HERRERA MORILLAS, J. Teoria y nuevos escenarios de la biblioteconomía. Buenos Aires: Alfagrama, 2006.

SPEDALIERI, G. (2007). Catalogación de monografias impresas. Buenos Aires: Alfagrama.

TAYLOR, Arlene.(1992). Introduction to Cataloging and Classification. Englewood: Libraries Unlimited. 